Check for updates

Cite this: RSC Adv., 2018, 8, 31237

Received 23rd July 2018

Accepted 29th August 2018

DOI: $10.1039 / \mathrm{c} 8 \mathrm{ra06232c}$

rsc.li/rsc-advances

\section{Exploring organic photosensitizers based on hemicyanine derivatives: a sustainable approach for preparation of amide linkages $\uparrow$}

\author{
Harnimarta Deol, Manoj Kumar and Vandana Bhalla (iD *
}

\begin{abstract}
Hemicyanine derivatives $\mathrm{C} 1-\mathrm{C} 4$ have been synthesized and show strong absorption in the visible region, good water solubility, efficient intersystem crossing, a high singlet oxygen quantum yield and high ability to transport electrons from the donor to acceptor. These hemicyanine derivatives were utilized as photocatalysts in additive/base free oxidative amidation of aromatic aldehydes in mixed aqueous media under visible light irradiation at low catalytic loading. The hemicyanine derivative C4 exhibited recyclability upto four cycles and reusability upto five cycles in oxidative amidation of aromatic aldehydes. Among all the hemicyanine derivatives, C4 shows a high photocatalytic efficiency due to a high singlet oxygen quantum yield. All the mechanism investigations showed involvement of reactive oxygen species generated by the organic triplet photosensitizer based on hemicyanine derivative for carrying out oxidative amidation of aldehyde. Our results will encourage the design of new "metal free" organic photosensitizers and their application in photocatalysis.
\end{abstract}

\section{Introduction}

The amide linkage is a structural backbone of proteins and peptides. These linkages are also predominant in medicinally important compounds, natural products and materials having industrial applications. The reason behind the high prevalence of the amide linkage is its high stability, polarity and conformational diversity. ${ }^{1}$ Conventional protocols for the preparation of amide bonds generate a significant amount of chemical waste/side products and hence have poor atom economy. ${ }^{2}$ Thus, a lot of efforts are being devoted to making the preparation of the amide linkage more 'green'. One of the best approache to achieve the targets of green synthesis is to carry out the reaction in the presence of some catalytic species. The catalysts are special as they influence the kinetics of the reaction positively, provide an alternative pathway to the reaction, and themselves remain unchanged. To date, a variety of reaction protocols centred around various catalytic systems based on transition metals $^{2,3}$ and $\mathrm{N}$-heterocyclic carbenes ${ }^{4}$ have been reported for amide bond formation. The majority of these catalyzed synthetic protocols require the presence of an oxidant, strong thermal conditions and an inert atmosphere which in fact reduces the economic and environmental advantages of the

Department of Chemistry, UGC Sponsored Centre for Advanced Studies-II, Guru Nanak Dev University, Amritsar 143005, Punjab, India. E-mail:vanmanan@yahoo.co.in $\dagger$ Electronic supplementary information (ESI) available: The contents of the ESI section include ${ }^{1} \mathrm{H},{ }^{13} \mathrm{C}$ NMR and ESI-MS spectra of compounds; UV-vis and fluorescence studies; time resolved fluorescence spectroscopy. See DOI: 10.1039/c8ra06232c strategy. These limitations have been overcome by utilization of "photosensitizers" as the photocatalysts for the preparation of the amide bond via oxidative amidation of aldehydes. In this context, a variety of commercially available ruthenium/iridium metal based complexes ${ }^{1,5}$ and dyes have been utilized as photocatalysts. Very recently, palladium carbodicarbene complex ${ }^{6}$ has been utilized as photocatalyst for the construction of amide bond in organic reaction media under visible irradiation. The main limitations of this approach are the requirement of the toxic, costly and synthetically difficult organometallic complexes as the catalytic species. Further, high loading of these photocatalysts are essential in the reaction mixture to get the target compound in good yield. The reason behind necessity of high loading of photocatalyst is weak/moderate absorption of these materials in visible region. Unfortunately, structural modification of these photocatalysts to enhance their absorption in visible region is also difficult. To overcome these limitations, several organic triplet photosensitizers based on BODIPY, ${ }^{7}$ phenazinium ${ }^{8}$ and quinolizinium compounds ${ }^{9}$ scaffold have been synthesized and tested for the photocatalytic synthesis of amide bonds (Scheme 1). In comparison to ruthenium/iridium heavy metal based complexes, these organic triplet photosensitizers absorb strongly in visible region and have long life time of the triplet excited state. Most of these photocatalytic systems are themselves capable of activating $\mathrm{O}_{2}$ under light irradiation and hence external oxidant is not needed. However, the economic/environmental advantages of these approaches are decreased due to their multistep synthetic routes, tedious purification and also the presence of boron as heavy atom (in BODIPY based catalytic system). Further, most of 


\section{Palladium carbodicarbene complex as photocatalyst ${ }^{6}$}

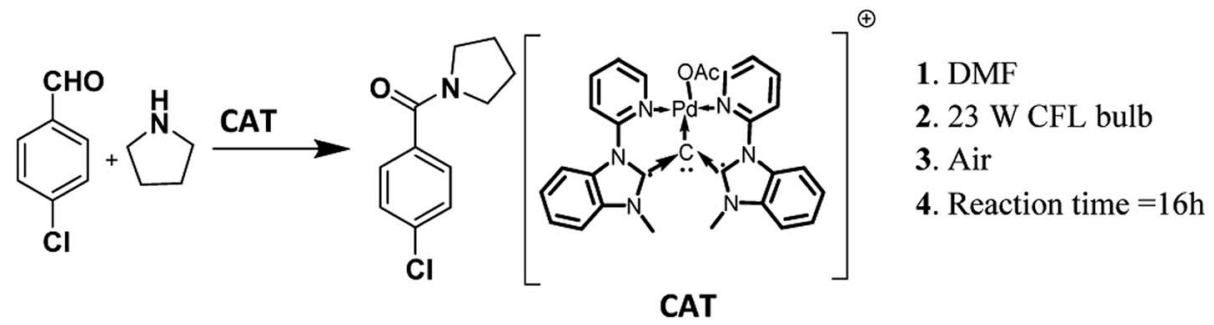

\section{BODIPY as photocatalyst ${ }^{7}$}

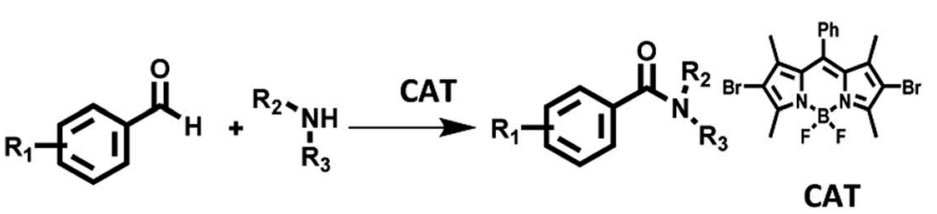

CAT
1. Dioxane

2. Blue LED

3. Air/rt

4. BHT additive

5. Reaction time $=12 \mathrm{~h}$

\section{Phenazinium salt as photocatalyst ${ }^{8}$}

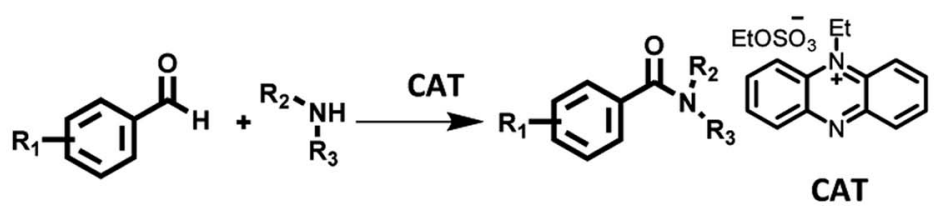

1. THF inhibitor free

2. $24 \mathrm{~W}$ house hold lamp

3. Air

4. Reaction time $=16 \mathrm{~h}$

\section{Quinolizinium compounds as photocatalyst ${ }^{9}$}

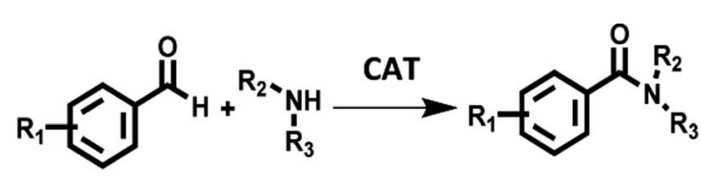

This Work

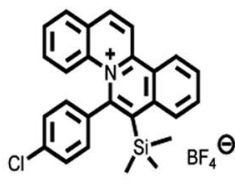

CAT
1. ACN

2. Blue LED

3. $\mathrm{Na}_{2} \mathrm{CO}_{3}$ as base

4. Air/rt

5. Reaction time $=48 \mathrm{~h}$

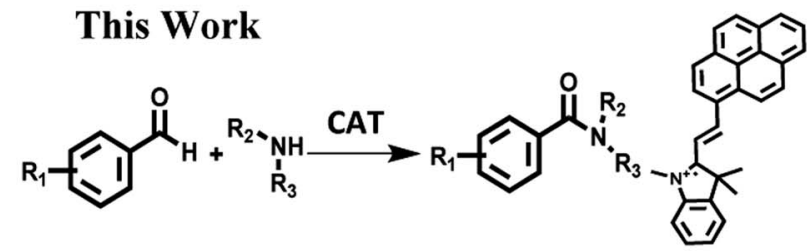

CAT
1. DMSO: $\mathrm{H}_{2} \mathrm{O}(1: 1)$

2. Tungsten bulb

3. Air/rt

4. Base/Additive free

5. Reaction time $=12 \mathrm{~h}$

6. Low catalytic loading ( $1 \mathrm{~mol} \%)$

7. Reusability

8. Recyclability

9. Dip strip as a portable catalyst

Scheme 1 Oxidative amidation of aromatic aldehyde.

these systems work only in organic media and in some cases the desired product is furnished in decent yield only in the presence of blue LED as source of radiation which again decreases the economic and environmental advantages of the strategy. Thus, development of an efficient photocatalyst/organic triplet photosensitizer which could work efficiently under visible light irradiation in base/additive free conditions in aqueous media is still a challenge.

Our research work involves the development of new photocatalytic systems for harvesting solar radiations for pursuing various organic transformations. ${ }^{10}$ In continuation of our efforts in this direction, we were then interested to develop a dye based photocatalytic system for amide bond formation via oxidative amidation of aromatic aldehydes in aqueous media under ecofriendly conditions. For preparation of such photocatalytic system, hemicyanine dye is scaffold of our choice due to its strong absorption in visible region, good water compatibility, straightforward synthetic route, ease of modification, long lived triplet excited state and its ability to generate reactive oxygen species. With these unique photophysical and photochemical properties hemicyanine dyes have multidisciplinary applications in various fields such as photodynamic therapy (PDT), 
bioimaging, construction of dye sensitized organic solar cells and pharmaceutical industry etc. $^{\mathbf{1 1}}$

Recently, from our lab we reported a hemicyanine derivative $\mathbf{C 1}$ which emits in NIR region and showed selective and sensitive response towards HSA in aqueous media. ${ }^{12}$ As a test of our hypothesis, we planned to the examine the photocatalytic efficiency of NIR active hemicyanine derivative C1 in additive/base free oxidative amidation of aromatic aldehydes in mixed aqueous media under visible light irradiation. To our delight, the reaction worked well and the desired product was obtained in $72 \%$ yield (vide infra). Encouraged by this result, we plan to synthesized a series of photocatalysts (C1-C4) based on hemicyanine dye and examine their efficiency in the oxidative amidation reaction. These photocatalysts based on hemicyanine dye were prepared by simple condensation of various aldehydes with 1,2,3,3-tetramethyl- $3 H$-indol-1-ium in ethanol. The photocatalytic activity of these hemicyanine derivatives were examined in additive/base free oxidative amidation of aromatic aldehydes under aerial conditions in aqueous media using tungsten filament bulb as the irradiation source. Amazingly, among all the synthesized derivatives, C4 having pyrene as donor unit exhibited high efficiency and furnished the target compound in good yield. The hemicyanine derivative $\mathbf{C 4}$ exhibited recyclability upto four cycles and reusability upto five cycles in oxidative amidation of aromatic aldehyde reaction. The high efficiency of derivative C4 may be attributed to its strong absorption in visible region, good water solubility, high singlet oxygen quantum yield $\left(\Phi_{\Delta}=\right.$ 0.85 ) and efficient intersystem crossing. The work being presented in this manuscript demonstrates the utilization of hemicyanine derivatives as efficient photocatalysts for oxidative amidation of aldehydes under solar radiation and under solvent free conditions. Unprecedented, this is the first report regarding utilization of hemicyanine derivatives as organic photosensitizer in photocatalysis under mild condition (aqueous media, base/additive free visible light and aerial conditions, low catalytic loading). Further, the 'dip strip' as portable catalytic system was prepared by dipping filter paper strip into the solution of derivative $\mathbf{C 4}$ for carrying out oxidative amidation of aromatic aldehyde under visible light irradiation.

\section{Results and discussion}

\section{Synthesis of the photosensitizers based on hemicyanine derivatives}

Derivatives C1, C3 and C4 were synthesized by following the procedure reported in literature (Fig. S40-S44 in ESI $\dagger$ ). ${ }^{\mathbf{1 2 - 1 4}}$ Derivative $\mathbf{C} 2$ was synthesized by condensation of 1,2,3,3-tetramethyl-3H-indol-1-ium with 2,7-azaindole-3-carboxaldehyde in ethanol (Scheme 2). ${ }^{15}$ The structure of $\mathbf{C} 2$ was characterized by various spectroscopic and analytical studies (Fig. S37-S39 in $\mathrm{ESI} \dagger)$.

\section{Photophysical properties}

The UV-vis and fluorescence studies of derivatives C1-C4 were examined in DMSO : $\mathrm{H}_{2} \mathrm{O}(1: 1)$. The results were summarized in Table 1 which clearly show that derivatives C1, C3 and C4 exhibit absorption/emission at longer wavelength in comparison to that of derivative $\mathbf{C 2}$. The comparatively red shifted emission in case of derivatives $\mathbf{C 1}, \mathbf{C} 3$ and $\mathbf{C 4}$ may be attributed to the presence of relatively stronger donor units and extended conjugation which strongly facilitates the intramolecular

Table 1 Photophysical parameters of photosensitizers based on hemicyanine derivatives $^{a}$

\begin{tabular}{lllllll}
\hline Photosensitizer & $\lambda_{\text {abs }}(\mathrm{nm})$ & $\varepsilon$ & $\lambda_{\mathrm{em}}(\mathrm{nm})$ & $\Phi_{\mathrm{F}}$ & $\tau_{\text {avg }}(\mathrm{ns})$ & $\Phi_{\Delta}$ \\
\hline C1 & 587 & 33000 & 686 & 0.04 & 0.78 & 0.70 \\
C2 & 457 & 25000 & 511 & 0.25 & 0.85 & $\mathrm{a}$ \\
C3 & 540 & 60000 & 557 & 0.20 & 0.8 & $\mathrm{a}$ \\
C4 & 500 & 32000 & $560 / 610$ & 0.03 & 0.50 & 0.85
\end{tabular}

${ }^{a} \varepsilon=$ molar extinction coefficient $\mathrm{M}^{-1} \mathrm{~cm}^{-1}, \Phi_{\mathrm{F}}=$ fluorescence quantum yield, $\tau_{\text {avg }}=$ average lifetime of singlet state, $\Phi_{\Delta}=$ singlet oxygen quantum yield, $\mathrm{a}=$ could not be detected.

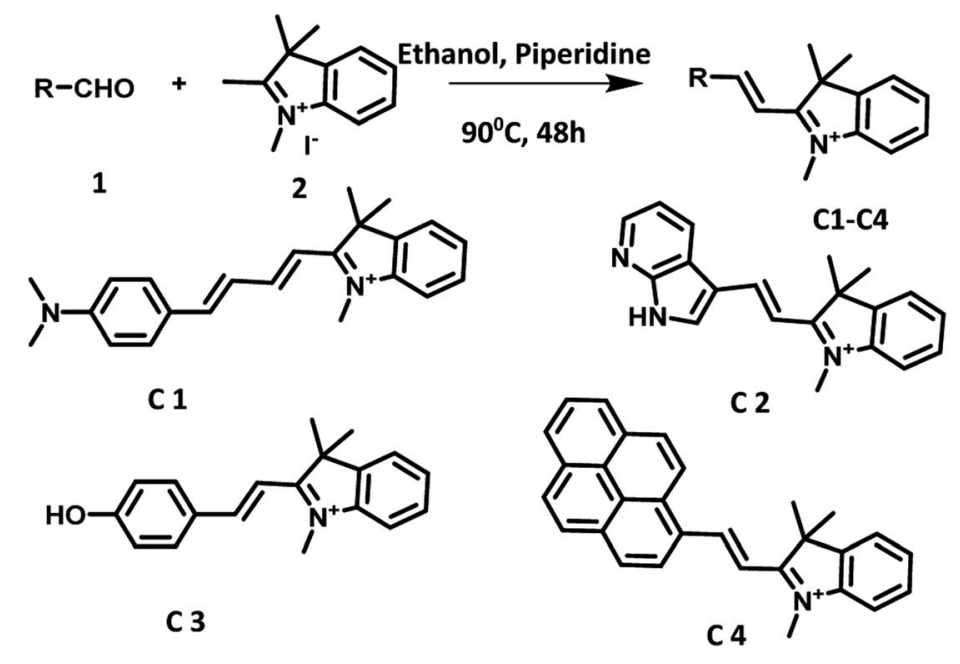

Scheme 2 Synthesis of photocatalysts based on hemicyanine derivatives (C1-C4). 
charge transfer in these systems (Fig. S1 and S2 in ESI + ). Further, the singlet state lifetime of the derivatives C1-C4 were evaluated using the time resolved fluorescence spectroscopy (Fig. S3-S6 in ESI†). In all the derivatives, life time of singlet state was found to be less than $1 \mathrm{~ns}$, thus, long lifetime of their respective triplet state is expected. Derivatives C2 and C3 showed decent quantum yields but in case of derivatives $\mathbf{C} \mathbf{1}$ and C4 the low quantum yields were determined (Table 1). The low quantum yields in case of derivatives $\mathbf{C} \mathbf{1}$ and $\mathbf{C 4}$ further indicate efficient intersystem crossing (ISC) form singlet to triplet excited state in these systems, as a result of which a highly stable/long lived triplet excited state is expected in these derivatives. Further we calculated the singlet oxygen quantum yields for derivatives $\mathbf{C 1}$ and $\mathbf{C 4}$ using 1,3-diphenylisobenzofuran (DPBF) a known ${ }^{1} \mathrm{O}_{2}$ quencher (Fig. $\mathrm{S} 7$ and $\mathrm{S} 8$ in ESI $\dagger$ ). ${ }^{16}$ Both the derivatives showed good efficiency for generation of singlet oxygen (Table 1).

Further, we checked the photostability of hemicyanine derivatives C1-C4 by irradiating their oxygen saturated solutions in mixed aqueous media for $36 \mathrm{~h}$ (Fig. S9-S12 in ESI $\dagger$ ). The derivatives C1-C3 showed good photostability even after continuous irradiation for $36 \mathrm{~h}$. However, in case of a derivative C4, 30\% decrease in absorbance intensity was observed after $36 \mathrm{~h}$.

In the next part of our work, we planned to examine the potential of photosensitizer based on hemicyanine derivative to transport electron from donor to acceptor unit which is key step in photocatalysis. For this we chose three component system consisting of methyl viologen $\left(\mathrm{MV}^{2+}\right)$ as electron acceptor, triethanolamine (TEOA) as sacrificial donor and hemicyanine derivative as the photosensitizer. We observed that upon addition of TEOA in solution of derivative $\mathbf{C 4}$, the band at $500 \mathrm{~nm}$ due to derivative $\mathbf{C 4}$ rapidly decreased along with red shift of $50 \mathrm{~nm}$. These observations indicate a strong interaction between sacrificial donor and derivative C4. Afterwards, we added methyl viologen $\left(\mathrm{MV}^{2+}\right)$ and upon exposure to room light under inert atmosphere within 2 min colour of solution changed from light pink to green. Further, within next 6 min colour of solution changed to dark blue. The whole event was monitored by UV-vis spectroscopy. The UV-vis spectra showed presence of two new bands at $\lambda=395$ and $603 \mathrm{~nm}$ which gradually increased upon exposure to room light (Fig. 1A). These two bands correspond to the reduced species $\mathrm{MV}^{++}$of methyl viologen. On the basis of these results, we proposed that derivative $\mathbf{C 4}^{*}$ reductively quenched by sacrificial donor and to form $\mathbf{C 4}^{*-}$, then $\mathbf{C 4}^{*-}$ transports electron to $\mathrm{MV}^{2+}$ and reduced it (Fig. 1B). ${ }^{17}$ We also performed the same experiment with other hemicyanine derivatives (C1-C3), the colour of solution gradually changed from green to blue (Fig. S13-S15 in ESI†). All above results showed that hemicyanine derivatives work as photosensitizers which transport the electron from sacrificial donor to acceptor under visible light.

\section{Photocatalytic amidation of aromatic aldehyde}

After examining the photophysical properties of derivatives C1C4, our initial investigation focused on the direct oxidative

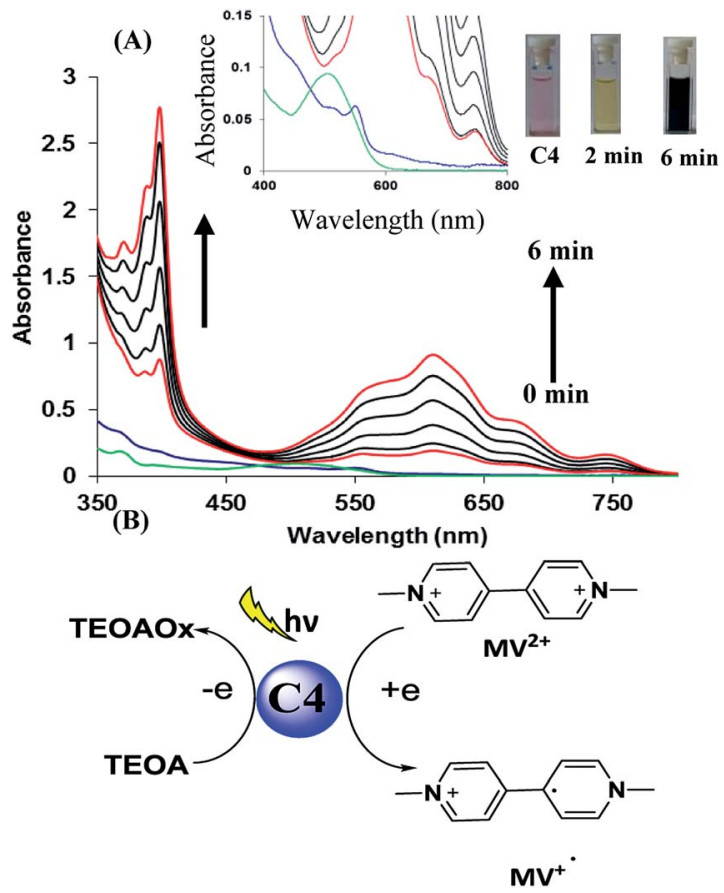

Fig. 1 (A) Absorption spectral changes of derivative $\mathrm{C} 4(0.02 \mathrm{mM})$ in presence of $\mathrm{MV}^{2+}(0.2 \mathrm{mM})$ and TEOA $(50 \mathrm{mM})$ in DMSO under room light and inert atmosphere. Inset: absorption spectral changes upon addition of TEOA in solution of derivative $\mathrm{C} 4$ and photograph of solution before and after 6 min. (B) Derivative C4 acts mediator and transport the electron from sacrificial donor to acceptor under visible light.

amidation of aromatic aldehyde under visible light irradiation. The reaction between 4-nitrobenzaldehyde (1a, 1.0 equiv.) and pyrrolidine (2a, 3.0 equiv.) in DMSO : $\mathrm{H}_{2} \mathrm{O}$ solvent mixture under the irradiation of tungsten filament bulb at room temperature was chosen as the model reaction. Among all the derivatives, we chose derivative $\mathbf{C 4}$ as a photocatalyst for carrying out model reaction due to its high singlet oxygen quantum yield and high efficiency to transport the electron from donor to acceptor. To our delight in presence of $1 \mathrm{~mol} \%$ of derivative $\mathbf{C 4}$, the reaction was completed in $12 \mathrm{~h}$ and the desired product was obtained in $82 \%$ yield (Table 2, entry 1 ). Next, we screened different solvents such as THF, MeCN, DMF, dioxane, DMSO and water (Table 2, entries 2-7) as the reaction media for the transformation. The desired product was obtained in comparable yields in case of DMSO and DMSO : $\mathrm{H}_{2} \mathrm{O}$ mixture and much lower yield of product was obtained in water. We chose DMSO : $\mathrm{H}_{2} \mathrm{O}(1: 1)$ as the reaction media for carrying out further transformations. Further, we also examined the influence of the presence/absence of base in the oxidative amidation of aromatic aldehyde under visible light irradiation (Table 2, entry 8). The presence or absence of base did not influence the yield of the desired product. Further, in the absence of a hemicyanine based photosensitizer or in absence of visible light source, the reaction did not proceed (Table 2, entries 9 and 10). The desired product was obtained in $10 \%$ yield when model reaction was setup under inert atmosphere (Table 2, entry 11). This result emphasized that aerial 


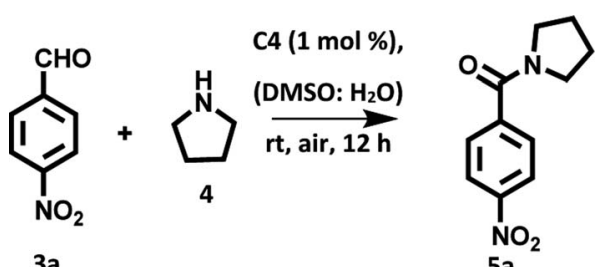

$3 a$

\begin{tabular}{|c|c|c|c|c|c|}
\hline Entry & Photosensitizer & Additive & Solvent & Time & Yield \\
\hline 1 & C4 & - & DMSO $: \mathrm{H}_{2} \mathrm{O}(1: 1)$ & $12 \mathrm{~h}$ & $82 \%$ \\
\hline 2 & C4 & - & DMSO & $12 \mathrm{~h}$ & $80 \%$ \\
\hline 3 & C4 & - & DMF & $12 \mathrm{~h}$ & $15 \%$ \\
\hline 4 & C4 & - & Dioxane & & $30 \%$ \\
\hline 5 & C4 & - & THF & $12 \mathrm{~h}$ & $55 \%$ \\
\hline 6 & C4 & - & $\mathrm{MeCN}$ & $12 \mathrm{~h}$ & $60 \%$ \\
\hline 7 & C4 & - & Water & $12 \mathrm{~h}$ & $30 \%$ \\
\hline 8 & C4 & $\mathrm{K}_{2} \mathrm{CO}_{3}$ as base & DMSO $: \mathrm{H}_{2} \mathrm{O}(1: 1)$ & $12 \mathrm{~h}$ & $82 \%$ \\
\hline $9^{a}$ & - & - & DMSO $: \mathrm{H}_{2} \mathrm{O}(1: 1)$ & $12 \mathrm{~h}$ & $0 \%$ \\
\hline $10^{b}$ & C4 & - & DMSO $: \mathrm{H}_{2} \mathrm{O}(1: 1)$ & $12 \mathrm{~h}$ & 0 \\
\hline $11^{c}$ & C4 & - & DMSO $: \mathrm{H}_{2} \mathrm{O}(1: 1)$ & $12 \mathrm{~h}$ & $10 \%$ \\
\hline $12^{d}$ & C4 & - & DMSO : $\mathrm{H}_{2} \mathrm{O}(1: 1)$ & $12 \mathrm{~h}$ & $60 \%$ \\
\hline 13 & C4 & $\mathrm{H}_{2} \mathrm{O}_{2}$ & DMSO $: \mathrm{H}_{2} \mathrm{O}(1: 1)$ & $12 \mathrm{~h}$ & $88 \%$ \\
\hline 14 & C4 & BHT & DMSO $: \mathrm{H}_{2} \mathrm{O}(1: 1)$ & $12 \mathrm{~h}$ & $85 \%$ \\
\hline 15 & C1 & - & DMSO $: \mathrm{H}_{2} \mathrm{O}(1: 1)$ & $20 \mathrm{~h}$ & $72 \%$ \\
\hline 16 & C2 & - & DMSO $: \mathrm{H}_{2} \mathrm{O}(1: 1)$ & $20 \mathrm{~h}$ & $50 \%$ \\
\hline 17 & $\mathrm{C} 3$ & - & DMSO $: \mathrm{H}_{2} \mathrm{O}(1: 1)$ & $16 \mathrm{~h}$ & $75 \%$ \\
\hline
\end{tabular}

conditions promote the oxidative amidation of aromatic aldehyde under visible light irradiation in presence of photocatalyst based on hemicyanine derivative. Further, we examined the effect of increase in the catalytic loading on the reaction kinetics and efficiency of the reaction. The desired product was obtained in lower yield $(60 \%)$ (Table 2, entry 12) upon increasing the catalytic loading from $1 \mathrm{~mol} \%$ to $4 \mathrm{~mol} \%$, which may be attributed to the increase in the colour intensity of the solution that hindered the passage of radiations. ${ }^{8}$ We also examined the effect of the presence of external oxidant such as $\mathrm{H}_{2} \mathrm{O}_{2}$ on the reaction kinetics. As expected, the reaction was accelerated and the desired product was furnished in $88 \%$ yield after $8 \mathrm{~h}$ (Table 2 , entry 13). Further, we also checked the effect of BHT as additive in the oxidative amidation of aldehyde under visible light irradiation. However, no significant effect on the yield and rate of reaction was observed (Table 2, entry 14).

Under these optimized reaction conditions, we also examined the photocatalytic activity of other $\mathbf{C} 1, \mathbf{C} 2$ and $\mathbf{C} 3$ hemicyanine derivatives in oxidative amidation of aldehyde. The derivatives C1, C2 furnished the desired product in $72 \%$ and $50 \%$ yields, respectively after $20 \mathrm{~h}$. On the other hand upon using $\mathbf{C} 3$ as the photocatalyst, the desired product was obtained in $75 \%$ yield after $16 \mathrm{~h}$ (Table 2, entries 15-17). Among all the derivatives $\mathbf{C 4}$ showed high photocatalytic activity due to its maximum efficiency for generation of singlet oxygen $\left(\Phi_{\Delta}=0.85\right)$ and high ability to transport the electron as compared to other hemicyanine derivatives. In comparison to the off shelf photosensitizers such as $\mathrm{Ru}(\text { phen })_{3} \mathrm{Cl}_{2}, \operatorname{Ir}(\mathrm{dtbpy})(\mathrm{ppy})_{2} \mathrm{PF}_{6}$, Rhodamine B, Methylene blue and Alizarin red S etc. (Table S1 in ESI $\dagger$ ), the photosensitizers based on hemicyanine derivatives worked well in the oxidative amidation of aldehyde under visible light irradiation.

In order to get more insight into the efficiency of derivative C4, we examined the photophysical properties of derivative $\mathbf{C 4}$ in presence of pyrrolidine. The solution of derivative $\mathbf{C 4}$ in DMSO showed emission bands at 560/610 nm. Upon addition of $0.1 \mathrm{mmol}$ of pyrrolidine to this solution, the intensity of emission bands at 560/610 nm were quenched completely (Fig. S16 in $\operatorname{ESI} \dagger$ ). On the other hand, higher equivalents of pyrrolidine were required in case of derivatives C1-C3 (Fig. S17-S19 in ESI†). These studies supported the high efficiency of electron transfer between pyrrolidine and excited state of derivative $\mathbf{C 4}$. $^{7}$

With the optimized reaction conditions in hand, a variety of aromatic aldehydes were investigated to illustrate the photocatalytic efficiency and scope of the photocatalyst based on hemicyanine derivative $\mathbf{C 4}$ under visible light irradiation and aerial conditions. Aromatic aldehydes bearing electron withdrawing groups and electron donating groups furnished the desired products in good yields (Scheme 3).

Furthermore, we examined the substrate scope with respect to amines having six membered rings such as morpholine and piperidine. Amazingly, in presence of these amines as the reaction partner the desired products were obtained in good yields (Scheme 4). 


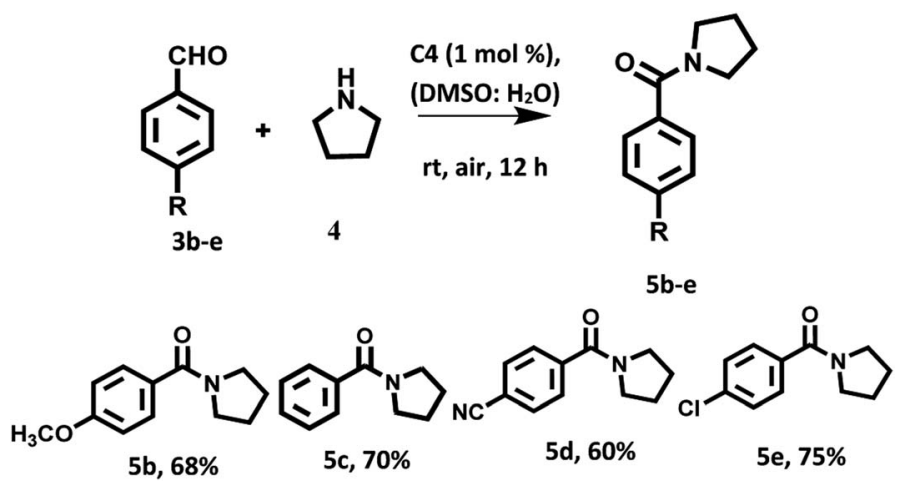

Scheme 3 Oxidation amidation of substituted aromatic aldehydes with pyrrolidine using photocatalyst based on hemicyanine derivative C4 under irradiation of visible light at room temperature and aerial conditions.

We also investigated the oxidative amidation of 4-nitrobenzaldehyde (1a, 1.0 equiv.) with pyrrolidine (2a, 3.0 equiv.) under solvent free conditions using of $1.0 \mathrm{~mol} \%$ of $\mathbf{C} 4$ under the irradiation of tungsten filament bulb at room temperature (Scheme 5). To our pleasure, the desired product was obtained in $70 \%$ yield after $20 \mathrm{~h}$. On the other hand the reaction between 4-methoxybenzaldehyde (1a, 1.0 equiv.) and pyrrolidine (2a, 3.0 equiv.) under solvent free conditions using $1 \mathrm{~mol} \%$ of $\mathbf{C 4}$ produced the desired product in $60 \%$ yield after $36 \mathrm{~h}$. All these studies highlighted the utility of photocatalyst in very mild conditions.

To further demonstrate the practical application of photocatalyst based on hemicyanine derivative for preparation of amides, we carried out reaction between 4-nitrobenzaldehyde (1a, 1.0 equiv.) and pyrrolidine (2a, 3.0 equiv.) in DMSO : $\mathrm{H}_{2} \mathrm{O}$ (1:1) using of $\mathbf{C 4}(1 \mathrm{~mol} \%)$ as photosensitizer one at $0.2 \mathrm{mmol}$ and another one at gram scale in presence of solar radiations (Fig. S20 in ESI $\uparrow$ ). Remarkably, after 8 and 36 h, the desired product was isolated in $88 \%$ and $70 \%$ yields respectively (Scheme 6). These results showed that $\mathbf{C 4}$ found to be more efficient in presence of solar light, which indicate that solar light accelerate reaction rate upto great extent than tungsten bulb using as irradiation source.

To determine the recyclability of photocatalytic system, we chose reaction between of 4-nitrobenzaldehyde (1a, 1.0 equiv.) and pyrrolidine (2a, 3.0 equiv.) in DMSO $: \mathrm{H}_{2} \mathrm{O}(1: 1)$ as the model reaction using $1.0 \mathrm{~mol} \%$ of derivative $\mathbf{C 4}$ as photocatalyst. After completion of the reaction, solvent was evaporated under reduced pressure and the resulting residue was extracted with the ethyl acetate. The organic part was separated and washed with water, dried over $\mathrm{Na}_{2} \mathrm{SO}_{4}$ and concentrated under reduced pressure. The aqueous layer containing photosensitizer was concentrated under reduced pressure and reused for next cycle of oxidative amidation of aldehyde (Fig. S21 in ESI †). The same procedure was followed for four cycles. After the fourth cycle, the yield of the final product was reduced to $62 \%$ (Fig. 2).

Further, we examined the reusability of derivative $\mathbf{C 4}$ in oxidative amidation of aldehyde. A reusability experiment was carried out using $\mathbf{C 4}$ as a catalyst for the oxidative amidation of aldehyde under visible light irradiation. After each cycle, reactants were introduced into the solution of original catalytic<smiles>Cc1ccc(C(=O)N2CCCC2)cc1</smiles>

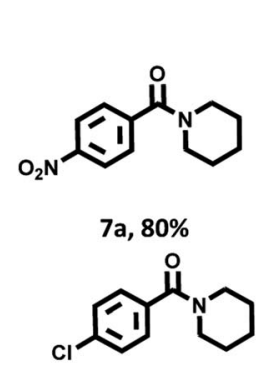

$7 e, 75 \%$
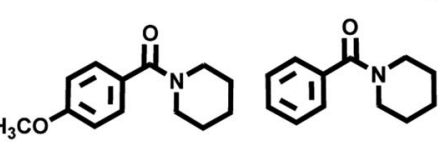

a-b

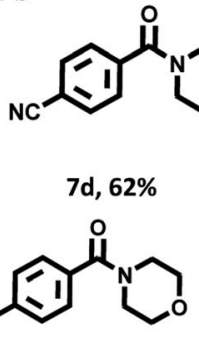

$8 b, 63 \%$

Scheme 4 Oxidation amidation of substituted aromatic aldehydes with piperidine/morpholine using photocatalyst based on hemicyanine derivative (C4) under irradiation of visible light at room temperature and aerial conditions. 


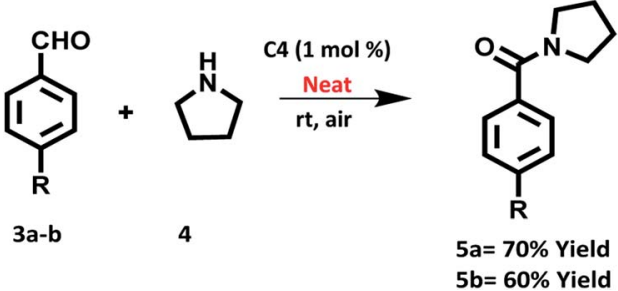

Scheme 5 Oxidation amidation of 4-nitrobenzaldehyde with pyrrolidine using photocatalyst based on hemicyanine derivative (C4) under irradiation of visible light at room temperature, neat and aerial conditions.

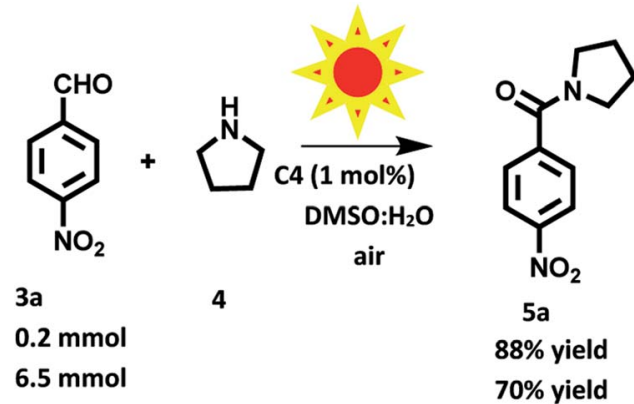

Scheme 6 Oxidation amidation of 4-nitrobenzaldehyde with pyrrolidine using photocatalyst based on hemicyanine derivative (C4) under solar radiation at room and aerial conditions.

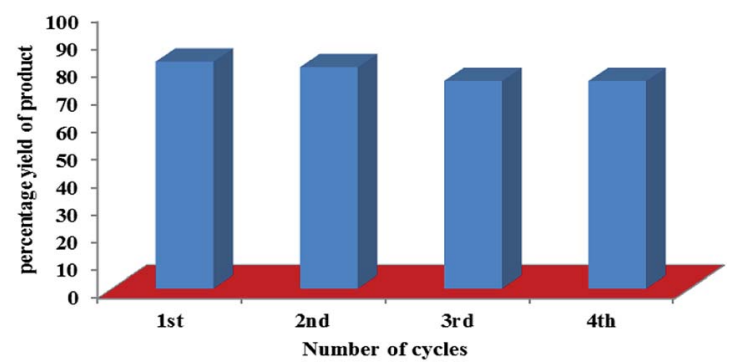

Fig. 2 Recyclability of photocatalyst based on hemicyanine derivative (C4) for carrying oxidative amidation of aromatic aldehyde under visible light irradiation.

system. The derivative $\mathbf{C 4}$ was successfully reused upto five cycles and no significant change in the yield of target product was observed (Fig. S22 in ESI†).<smiles>O=Cc1ccc([N+](=O)[O-])cc1</smiles>

3a

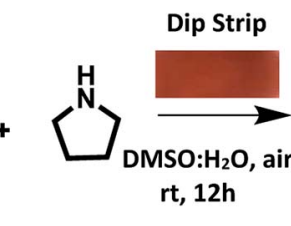

4

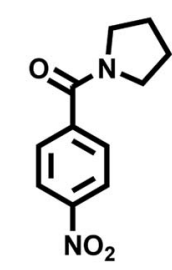

$5 a$

Yield $=80 \%$
Scheme 7 Oxidation amidation of 4-nitrobenzaldehyde with pyrrolidine using dip strip of derivative $\mathrm{C} 4$ under visible irradiation at room temperature and aerial conditions.

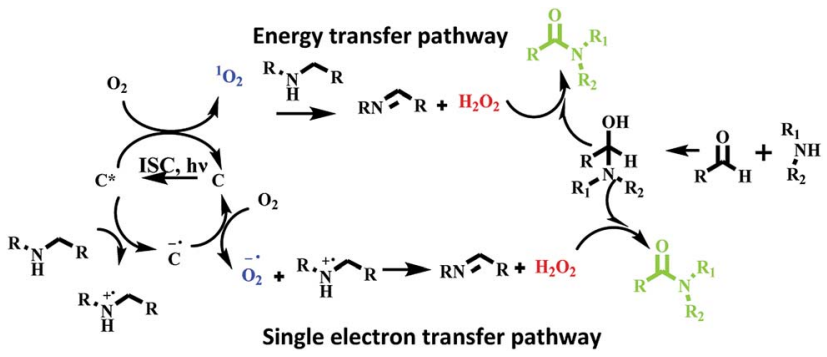

Scheme 8 Proposed reaction mechanism for oxidative amidation of aldehyde using photosensitizer C4 under visible light irradiation.

We also prepared 'dip strip' by dip coating a filter paper strip in solution of derivative C4 (Fig. S23 in ESI†). The prepared coated filter paper strip was used as portable catalytic system for carrying out oxidative amidation of aldehyde under visible light irradiation. To our pleasure the reaction was completed in $12 \mathrm{~h}$ with $80 \%$ yield of target product (Scheme 7).

\section{Mechanism studies}

We believe that oxidative amidation of aromatic aldehyde was mediated by reactive oxygen species generated by photosensitizer based on hemicyanine derivative in excited state. For generation of reactive oxygen species, two pathways are suggested. First, energy transfer pathway in which energy of triplet
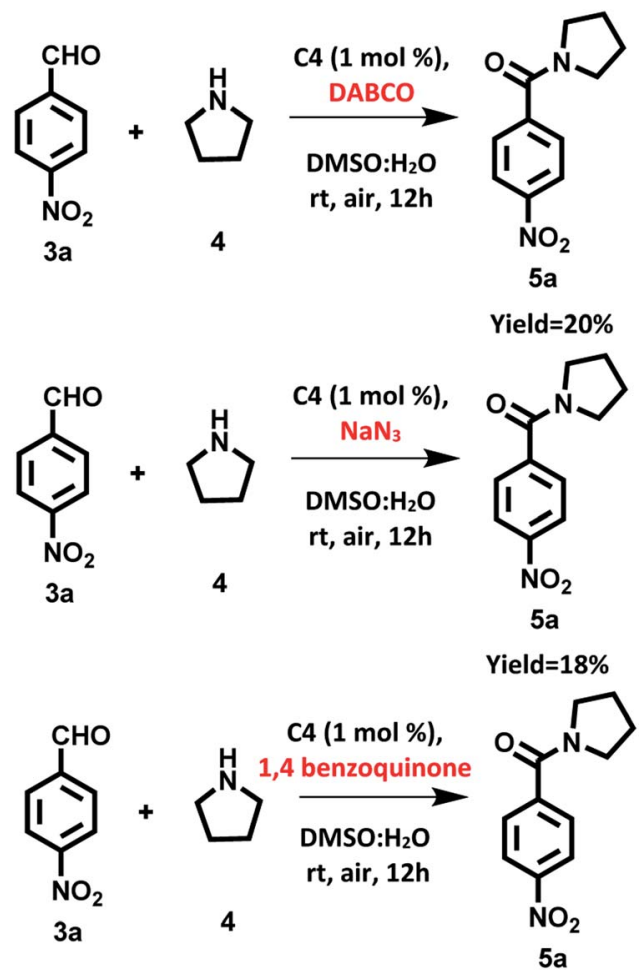

Yield $=\mathbf{2 2} \%$

Scheme 9 Oxidation amidation of 4-nitrobenzaldehyde with pyrrolidine in the presence of ROS quenchers using photocatalyst based on hemicyanine derivative (C4) under irradiation of visible light at room temperature and aerial conditions. 
excited state $\mathrm{C}^{*}$ transferred to dioxygen and singlet oxygen species was generated, which further reacts with amine to produce $\mathrm{H}_{2} \mathrm{O}_{2}$. The second proposed pathway is activation of dioxygen through a single electron transfer (SET) route to form the superoxide radical $\mathrm{O}_{2}{ }^{\cdot-}$ from the radical anion [C] ${ }^{\cdot-}$, which is generated by SET from the amine to the excited $\operatorname{state}^{3}[\mathrm{C}]^{*}$. Then, the active species $\mathrm{O}_{2}{ }^{--}$produced $\mathrm{H}_{2} \mathrm{O}_{2}$. The in situ generated $\mathrm{H}_{2} \mathrm{O}_{2}$ further oxidized $\alpha$-hydroxy amine to afford the amide (Scheme 8).

To check in situ generation of $\mathrm{H}_{2} \mathrm{O}_{2}$, we added $\mathrm{KI} / \mathrm{CH}_{3} \mathrm{COOH}$ to the reaction mixture and brown coloration was observed which confirms the presence of $\mathrm{H}_{2} \mathrm{O}_{2}$ in the reaction mixture (Fig. S24 in ESI $\dagger)^{7,16 c}$

To confirm our assumption regarding the involvement of singlet oxygen/superoxide in reaction, we carried out the model reaction in the presence of singlet oxygen scavenger such as DABCO (1,4-diazabicyclo[2.2.2]-octane), sodium azide and in presence of superoxide scavenger such as 1,4-benzoquinone. We found that the photocatalytic reaction was affected significantly and the desired product was obtained in $20 \%, 18 \%$ and $22 \%$ yields respectively (Scheme 9). These observations support that both ${ }^{1} \mathrm{O}_{2}$ and $\mathrm{O}_{2}{ }^{--}$are involved in the photocatalytic reaction.

\section{Conclusion}

Hemicyanine derivatives C1-C4 have been synthesized and utilized as photocatalysts in oxidative amidation of aromatic aldehydes under mild conditions. Among all the synthesized derivatives, $\mathbf{C 4}$ exhibited high efficiency which is attributed to its strong absorption in visible region, good water solubility, high singlet oxygen quantum yield $\left(\Phi_{\Delta}=0.85\right)$, efficient intersystem crossing and high ability to transport electron from donor to acceptor. All the mechanism investigations show participation of reactive oxygen species generated by triplet organic photosensitizer in oxidative amidation of aldehyde. The mild reaction conditions such as aerial conditions, aqueous media, low catalytic loading and visible light irradiation with broad substrate scope make this approach practically useful and eco-friendly for the synthesis of amide bond. Findings of this work will inspire the designing of new of "metal free" photosensitizers as photocatalyst in organic transformations.

\section{Experimental section}

\section{General experimental methods and materials ${ }^{18}$}

The general experimental methods, quantum yield calculations, and materials used are the same as those reported earlier by us.

UV-vis and fluorescence experiment. ${ }^{18}$ The stock solutions $\left(10^{-3} \mathrm{M}\right)$ of hemicyanine derivatives were prepared by dissolving $3.33 \mathrm{mg}, 3.02 \mathrm{mg}, 2.78 \mathrm{mg}$ and $3.86 \mathrm{mg}$ of compound C1, C2, C3 and C4 respectively in 10.0 mL of DMSO. 15.0 $\mu \mathrm{L}$ of this stock solution further diluted with add $1485 \mu \mathrm{L}$ of DMSO and $1500 \mu \mathrm{L}$ of water $(\mathrm{pH}=7.05)$ prepare $3.0 \mathrm{~mL}$ solution of derivative $\mathbf{C 1 - C 4}$ $(5.0 \mu \mathrm{M})$ and this solution was used for each UV-vis and fluorescence experiments.
Determination of singlet oxygen quantum yield. Singlet oxygen quantum yields of derivatives $\mathbf{C} 1$ and $\mathbf{C 4}$ were obtained by the measurement of quenching of DPBF in the presence of C1 and $\mathbf{C 4}$ as photosensitizers taking the reference of methylene blue under same conditions $\left(\Phi_{\Delta}=0.52\right)$ in DMSO as solvent. The irradiation was done in a fluorescence spectrophotometer equipped with a Xe lamp at the excitation wavelength of C1 and C2 for 50 min. UV-visible measurements were performed using UV-vis spectrophotometer. All the experiments were carried out at $25{ }^{\circ} \mathrm{C}$.

Synthesis of derivative C2. To a the mixture of 1,2,3,3-tetramethyl-3H-indol-1-ium (205 mg, $0.68 \mathrm{mmol}$ ) and 2, 7-azaindole3-carboxaldehyde (100 mg, $0.68 \mathrm{mmol}$ ) in anhydrous ethanol $(20.0 \mathrm{~mL})$ were added two drops of piperidine. Then the reaction mixture was refluxed for $48 \mathrm{~h}$ under inert atmosphere. After completion of reaction, red coloured solid was filtered and washed with ethanol (yield $=80 \%)$. ${ }^{1} \mathrm{H}$ NMR (300 MHz, DMSO$\left.d_{6}\right) \delta=13.29(\mathrm{~s}, 1 \mathrm{H}, \mathrm{NH}), 8.87-8.83(\mathrm{~m}, 2 \mathrm{H}), 8.74(\mathrm{~d}, J=15 \mathrm{~Hz}$, $2 \mathrm{H}), 8.54(\mathrm{~d}, J=6 \mathrm{~Hz}, 2 \mathrm{H}), 7.90(\mathrm{t}, J=7.5 \mathrm{~Hz}, 2 \mathrm{H}), 7.69-7.59(\mathrm{~m}$, 2H), 7.52-7.48 (m, 1H), 7.31 (d, J=15 Hz, 2H), 4.13 (s, 3H), 1.89 $(\mathrm{s}, 6 \mathrm{H}) .{ }^{13} \mathrm{C}$ NMR $\left(125 \mathrm{MHz}, \mathrm{DMSO}-d_{6}\right) \delta=181.15,151.00$, 148.90, 145.71, 143.14, 140.44, 130.52, 129.45, 123.16, 119.11, 114.41, 106.93, 56.50; mass, $m / z=302.16[\mathrm{M}+1]^{+}$.

General procedure for oxidative amidation of aromatic aldehyde using hemicyanine derivative under visible light irradiation

The mixture of aromatic aldehydes ( $0.2 \mathrm{mmol}, 1.0$ equiv.) and amine ( $0.6 \mathrm{mmol}, 3.0$ equiv.) in DMSO : $\mathrm{H}_{2} \mathrm{O}(1: 1)$ in presence of $\mathbf{C 4}(1 \mathrm{~mol} \%)$ as a photocatalyst was stirred at room temperature for $12 \mathrm{~h}$ under aerial condition and visible light irradiation (for products 5a, 6a, 8a, 7e, 5e), $20 \mathrm{~h}$ (for 5c, 5d, 7c, 7d) and $30 \mathrm{~h}$ (for substrate $\mathbf{5 b}, \mathbf{7 b}, \mathbf{8 b}$ ). After the completion of the reaction (monitored by TLC), organic part was extracted with EtOAc. The organic layer dried over anhydrous sodium sulphate and concentrate under reduced pressure. The crude was purified by column chromatography using EtOAc : hexane $(1: 1)$ to furnished the desired products. All the products were identified by ${ }^{1} \mathrm{H}$ NMR spectroscopy (Fig. S25-S36 in the ESI $\dagger$ ).

\section{Preparation of photocatalyst coated dip strip}

The filter paper strip was dipped in DMSO solution of C4 photocatalyst $(10.0 \mu \mathrm{M})$ for $1 \mathrm{~h}$. Afterwards, removed the filter paper and air dried. The dried filter paper strip has been used for carrying out the oxidative amidation of the aromatic aldehydes under visible light.

\section{Conflicts of interest}

There are no conflicts to declare.

\section{Acknowledgements}

V. B. is thankful to Science and Engineering Research Board (SERB), New Delhi (ref. no. EMR/2014/000149) for financial support. M. K. is thankful to Science and Engineering Research Board (SERB) (ref no. EMR/2016/003473). H. D. is thankful to 
UGC-BSR for Senior Research Fellowship (SRF). We are also thankful to UGC (New Delhi) for "University with Potential for Excellence" (UPE) project.

\section{Notes and references}

1 N. Iqbal and E. J. Cho, J. Org. Chem., 2016, 81, 1905.

2 (a) O. P. S. Patel, D. Anand, R. K. Maurya and P. P. Yadav, Green Chem., 2015, 17, 3728; (b) E. Valeur and M. Bradley, Chem. Soc. Rev., 2009, 38, 606; (c) J. R. Martinelli, T. P. Clark, D. A. Watson, R. H. Munday and S. L. Buchwald, Angew. Chem., Int. Ed., 2007, 46, 8460; (d) C. Gunanathan, Y. Ben-David and D. Milstein, Science, 2007, 317, 790; (e) L. U. Nordstrøm, H. Vogt and R. Madsen, J. Am. Chem. Soc., 2008, 130, 17672; $(f)$ D. Srimani, E. Balaraman, P. Hu, Y. Ben-David and D. Milstein, Adv. Synth. Catal., 2013, 355, 2525.

3 (a) B. Kang, Z. Fu and S. H. Hong, J. Am. Chem. Soc., 2013, 135, 11704; (b) C. Gunanathan and D. Milstein, Science, 2013, 341, 1229712; (c) C. Chen and S. H. Hong, Org. Biomol. Chem., 2011, 9, 20.

4 (a) S. De Sarkar and A. Studer, Org. Lett., 2010, 12, 1992; (b) J. W. Bode and S. S. Sohn, J. Am. Chem. Soc., 2007, 129, 13798; (c) H. U. Vora and T. Rovis, J. Am. Chem. Soc., 2007, 129, 13796; (d) L. Ta and H. Sunden, Chem. Commun., 2018, 54, 531.

5 J. Liu, Q. Liu, H. Yi, C. Qin, R. Bai, X. Qi, Y. Lan and A. Lei, Angew. Chem., Int. Ed., 2014, 53, 502.

6 Y.-C. Hsu, V. C.-C. Wang, K.-C. A. Yeung, C.-Y. Tsai, C.-C. Chang, B.-C. Lin, Y.-T. Chan, C.-P. Hsu, G. P. A. Yap, T. Jurca and T.-G. Ong, Angew. Chem., Int. Ed., 2018, 130, 4712.

7 X.-F. Wang, S.-Sh. Yu, C. Wang, D. Xue and J. Xiao, Org. Biomol. Chem., 2016, 14, 7028.

8 D. Leow, Org. Lett., 2014, 16, 5812.

9 J.-R. Deng, W.-C. Chan, N. C.-H. Lai, B. Yang, C.-S. Tsang, B. C.-B. Ko, S. L.-F. Chan and M.-K. Wong, Chem. Sci., 2017, 8, 7537.

10 (a) P. K. Walia, M. Kumar and V. Bhalla, ACS Omega, 2018, 3, 1983; (b) G. Singh, M. Kumar, K. Sharma and V. Bhalla, Green Chem., 2016, 18, 3278; (c) S. Kaur, M. Kumar and V. Bhalla, Green Chem., 2016, 18, 5870; (d) H. Deol, S. Pramanik, M. Kumar, I. A. Khan and V. Bhalla, ACS Catal., 2016, 6, 3771.

11 (a) C. T. Mapp, E. A. Owens, M. Henary and K. B. Grant, Bioorg. Med. Chem. Lett., 2014, 24, 214; (b) F. Shao, H. Yuan, L. Josephson, R. Weissleder and S. A. Hilderbrand, Dyes Pigm., 2011, 90, 119; (c) N. S. James, P. Joshi, T. Y. Ohulchanskyy, Y. Chen, W. Tabaczynski, F. Durrani, M. Shibata and R. K. Pandey, Eur. J. Med. Chem., 2016, 122, 770; (d) A. P. Gorka, R. R. Nani and M. J. Schnermann, Org. Biomol. Chem., 2015, 13, 7584; (e) S. J. J. Kwok, M. Choi, B. Bhayana, X. Zhang, C. Ran and S.-H. Yun, Sci. Rep, 2016, 6, 23866; (f) H. A. Shindy, M. A. El-Maghraby and F. M. Eissa, Dyes
Pigm., 2012, 92, 929; (g) H. A. Shindy, Rev. Roum. Chim., 2015, 60, 5; (h) H. A. Shindy, Eur. J. Mol. Biotechnol., 2016, 14, 158; (i) Q.-H. Yao, L. Shan, F.-Y. Li, D.-D. Yin and C.-H. Huang, New J. Chem., 2003, 27, 1277; (j) D. O. Mártire, W. Massad, H. Montejano, M. C. Gonzalez, P. Caregnato, L. S. Villata and N. A. García, Chem. Pap., 2014, 68, 1137; (k) X. Liu, G. Yang, L. Zhang, Z. Liu, Z. Cheng and X. Zhu, Nanoscale, 2016, 8, 15323; (l) S. Jenatsch, R. Hany, A. C. Véron, M. Neukom, S. Züfle, A. Borgschulte, B. Ruhstaller and F. Nüesch, J. Phys. Chem. $C, 2014,118,17036$; $(m)$ E. Stathatos and P. Lianos, Chem. Mater., 2001, 13, 3888; (n) M. S. A. Abdel-Mottaleb, M. M. S. Abdel-Mottaleb, H. S. Hafez and M. Saif, Int. J. Photoenergy, 2014, 2014, 1; (o) W. Wu, J. Hua, Y. Jin, W. Zhan and T. He, Photochem. Photobiol. Sci., 2008, 7, 63; (p) M. Cheng, X. Yang, J. Li, C. Chen, J. Zhao, Y. Wang and L. Sun, Chem.-Eur. J., 2012, 18, 16196; (q) Z.-S. Wang, F.-Y. Li and C.-H. Huang, J. Phys. Chem. B, 2001, 105, 9210; (r) O. Malinkiewicz, T. Grancha, A. Molina-Ontoria, A. Soriano, H. Brine and H. J. Bolink, Adv. Energy Mater., 2013, 3, 472; (s) W. Wu, F. Guo, J. Li, J. He and J. Hua, Synth. Met., 2010, 160, 1008; $(t)$ X. Ma, J. Hua, W. Wu, Y. Jin, F. Meng, W. Zhan and H. Tian, Tetrahedron, 2008, 64, 345.

12 S. I. Reja, I. A. Khan, V. Bhalla and M. Kumar, Chem. Commun., 2016, 52, 1182.

13 Y. Wu, J. Wang, F. Zeng, S. Huang, J. Huang, H. Xie, C. Yu and S. Wu, ACS Appl. Mater. Interfaces, 2016, 8, 1511.

14 J. Chang, Y. Lu, S. He, C. Liu, L. Zhaoab and X. Zeng, Chem. Commun., 2013, 49, 6259.

15 S. Wang, H. Xu, Q. Yang, Y. Song and Y. Li, RSC Adv., 2015, 5, 47990.

16 (a) J. Zhao, W. Wu, J. Sun and S. Guo, Chem. Soc. Rev., 2013, 42, 5323; (b) T. Yogo, Y. Urano, Y. Ishitsuka, F. Maniwa and T. Nagano, J. Am. Chem. Soc., 2005, 127, 12162; (c) L. Huang, J. Zhao, S. Guo, C. Zhang and J. Ma, J. Org. Chem., 2013, 78, 5627; (d) S. Guo, R. Tao and J. Zhao, RSC Adv., 2014, 4, 36131; (e) S. Mai, P. Marquetand and L. González, J. Phys. Chem. A, 2015, 119, 9524; (f) Y. Niko, S. Sasaki, K. Narushima, D. K. Sharma, M. Vacha and G. Konishi, J. Org. Chem., 2015, 80, 10794; $(g)$ H. Dinçalp, S. Kızılok and S. İcli, J. Fluoresc., 2014, 24, 917.

17 (a) S. D.-M. Islam, T. Konishi, M. Fujitsuka, O. Ito, Y. Nakamura and Y. Usui, Photochem. Photobiol., 2000, 71, 675; (b) Y. S. Park, S. Y. Um and K. B. Yoon, J. Am. Chem. Soc., 1999, 121, 3193; (c) M. Suzuki, C. C. Waraksa, T. E. Mallouk, H. Nakayama and K. Hanabusa, J. Phys. Chem. B, 2002, 106, 4227; (d) W.-W. Yong, H. Lu, H. Li, S. Wang and M.-T. Zhang, ACS Appl. Mater. Interfaces, 2018, 10, 10828; (e) J. Peon, X. Tan, J. D. Hoerner, C. Xia, Y. F. Luk and B. Kohler, J. Phys. Chem. A, 2001, 105, 5768.

18 S. Pramanik, V. Bhalla and M. Kumar, ACS Appl. Mater. Interfaces, 2014, 6, 5930. 